\title{
DESIGN OF A MICROGRIPPING SYSTEM WITH VISUAL AND FORCE FEEDBACK FOR MEMS APPLICATIONS
}

\author{
I. Giouroudi ${ }^{1}$, H. Hötzendorfer ${ }^{2}$, D. Andrijasevic ${ }^{1}$, M. Ferros ${ }^{2}$, W. Brenner ${ }^{1}$ \\ ${ }^{1}$ Institute of Sensor and Actuator Systems, Vienna University of Technology, Austria \\ ${ }^{2}$ Fundacion ROBOTIKER, Department of Design, Engineering and Manufacturing, Zamudio, Spain
}

Keywords: Microhandling, micromanipulation, visual servoing.

\begin{abstract}
This paper reports the design of a microgripping system with visual and force feedback for handling and assembly in microtechnology. The system consists of a microgripper, an electromagnetic microactuator, the Kleindiek Micromanipulator MM3A, a light microscope, a CCD camera and a computer. The microgripper is mounted on the Kleindiek Micromanipulator MM3A which has 3 degrees of freedom (two rotational axes, one linear axis), wide working range $\left(240^{\circ}\right.$ in the rotational axes, $12 \mathrm{~mm}$ in the linear axis) and subnanometer resolution. An external CCD camera provides images for obtaining the actual axis positions and by applying inverse kinematics the actual position of the manipulators end-effector is calculated so as to perform coarse positioning. A second CCD camera in combination with a light microscope provides sufficiently precise data to perform the actual manipulation tasks. Since the gripper is electromagnetically driven, the movement of the gripper tips is a continuous mapping of the applied current. The applied current can be correlated to the actual distance between the tips by calibration. In case of gripping an object, the tips are hindered to close to the full extent. The resulting gripping force depends on the actual distance changes.
\end{abstract}

\section{Introduction}

In recent years the need of miniaturization in many research fields such as biotechnology and nanotechnology and industrial fields such as microelectronics, MEMS, microsystem technologies etc has rapidly increased [1]. This has led to the development of microsystems with smaller components and increasingly complex microstructures. Consequently the need of new micromanipulation instruments and more sophisticated micromanipulation techniques with high accuracy and speed has as well increased [2, 3]. Therefore, micromanipulation has become a great technological challenge and the research in this field is receiving worldwide interest. The most challenging task and ultimate goal of this field is the development of computer assisted systems able to perform manipulation and assembly tasks in the micro world with the same accuracy and speed as it has already been achieved by robotics in the macro world. Great emphasis is given as well on the capability of the micromanipulation systems or micromanipulator robots to move over long distance, to manipulate different types of objects and to be functional in response to time without maintenance $[2,4]$.

Visual servoing is a rapidly maturing approach to the control of robot manipulators which is based on the visual perception of the robot/manipulator and the workpiece location. Specifically, visual servoing involves the use of one or more cameras and a computer vision system to control the position of the manipulator's end-effector (e.g. microgripper) relative to the workpiece as required by the performed task. It is a multi-disciplinary research area spanning computer vision, robotics, kinematics, dynamics, control and real-time systems [5].

In general, robots are known for their high flexibility, their applicability to different tasks and their speed and precision. However, compared to the human beings they lack the ability of seeing. Thus, the working environment has to adapt to the robots, as the robots are not able to adapt to the variable working conditions. These constraints in robot application led to the idea of providing robots with visual perception. Thus, yet in the early 70 s the term visual feedback was used to characterize robot control based on machine vision. Due to the lack of computation power and theoretical considerations open loop control was applied at the beginning. Machine Vision systems were quite slow and positional accuracy was low caused by the open loop manner of the robot control. The consequent improvements in vision algorithms and the increase of the computation power enabled the application of real closed loop position control of a robot manipulator being referred to as visual servoing since then. Several microrobotbased microhandling and microassembly stations have been proposed up to now in which handling and assembly is executed under multiple-view control techniques [4, 6-9]. Yet, human interaction is most of the times strongly required as unpredicted situations, such as noisy visual sensing information, may occur.

Taking these requirements into account a flexible, microgripping system with visual feedback has been designed, developed and tested aiming to automatize the function of a commercially available micromanipulator (Kleindiek Micromanipulator MM3A) and optimize the automation of pick-and-place tasks in the real environment. 
In the next section the design and the implementation of the microgripping system will be presented. The positioning and the gripping control of the developed system will also be discussed. Pick and place tasks will be reported in section 3 and section 4 concludes the paper.

\section{Design and Implementation}

The developed microgripping system consists of the Kleindiek Micromanipulator MM3A, a magnetic microgripper, a movable platform [10], a stereoscopic microscope in combination with a CCD camera, an additional external CCD camera, an image acquisition board (Imaq PCI1408) and CVI LabWindows/ IMAQ Vision software. Figure 1 shows a schematic of the system and Figure 2 shows a photo of the same system.

In particular, the Kleindiek micromanipulator has 3 degrees of freedom (two rotational axes, one linear axis), compact size (length of $65 \mathrm{~mm})$, a wide working range $\left(240^{\circ}\right.$ in the rotational axes, $12 \mathrm{~mm}$ in the linear axis) and a subnanometer resolution $(0,25 \mathrm{~nm}$ in the linear axis, $5 \mathrm{~nm}$ in the rotational axes). However, the micromanipulator does not possess positional encoders; therefore a feedback of the current position of the manipulator axes is not available. Moreover, the control unit is only able to move the robot stepwise. Line tracking is as well not available and hence the application in automated mode is not possible. In order to overcome these limitations visual feedback and inverse kinematics are applied.

As end effector an electromagnetically actuated microgripper is mounted on the micromanipulator. Electromagnetic actuation was chosen due to the fact that it is easier to control the displacement of the tips as well as the gripping force by controlling the applied current. Moreover, electromagnetic actuators are more reliable when operating in atmospheric conditions in comparison for example to electrostatic actuators (e.g dust might hinder their functionality), Finally, in case magnetic remenance occurs during the operation of the magnetic microgripper by changing the polarity the tips will open again and the component will be released without further complications.

By using the external CCD camera the position of the manipulators end-effector is acquired and by applying inverse kinematics the positions of the axes are obtained. Once the manipulator has reached the desired working position, which means that it is in the field of vision of the chosen microscope, the operation mode changes from coarse to fine positioning. The camera, which is directly connected to the microscope, provides the new input for the manipulator control.

The fact the microgripper is electromagnetically driven means that the movement of the microgripper tips is a continuous mapping of the applied current. This means that by knowing this current, the actual position of the tips can be calculated. In case of gripping an object, the tips will be hindered to close to the full extent. Comparing the actual position of the tips with the applied current the gripping force can be calculated. Allowing for force feedback, real time measurement of the microgripper tip aperture has to be performed. Machine
Vision algorithms can be implemented conducting this task. Due to the very simple geometry of the tips (see Fig. 3), simple pattern matching will lead to satisfying results and online measuring and hence force feedback can be achieved.

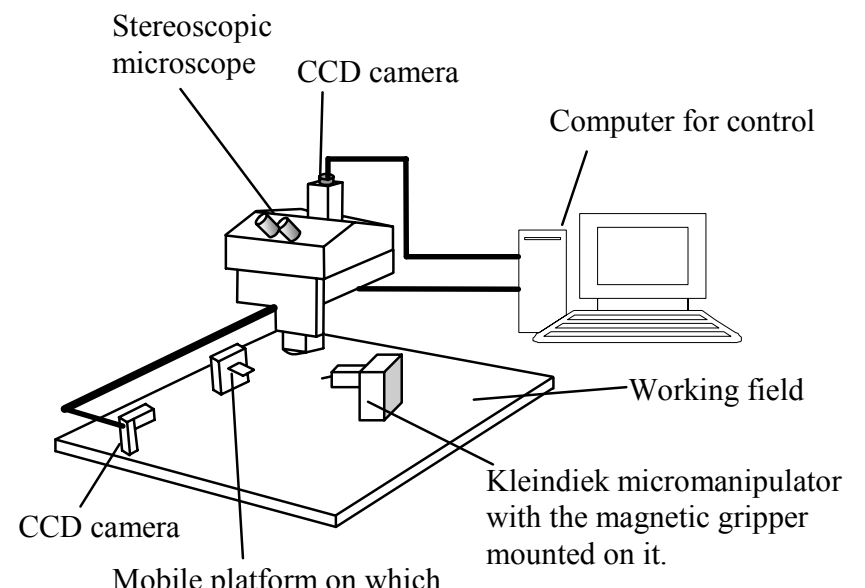
the microcomponents are placed

Figure 1: Visual feedback system.

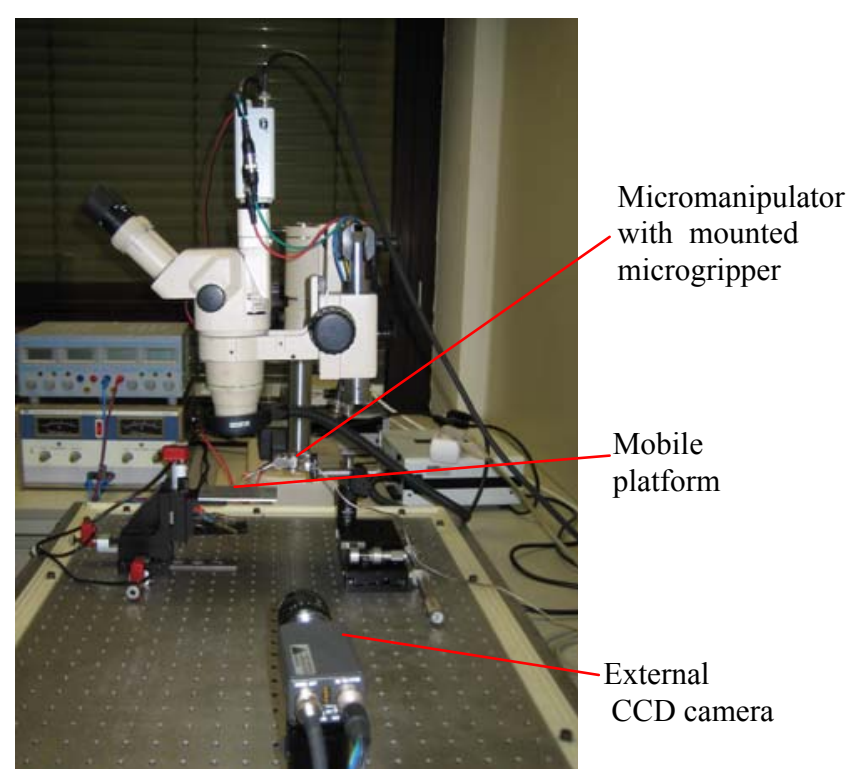

Figure 2: Photo of the whole visual feedback system.

\subsection{Microgripper}

The arms and tips of the microgripper are fabricated using an amorphous alloy which exhibits excellent soft magnetic and mechanical properties (VITROVAC 7505). Cold laser cutting technique (wavelength $\lambda=1064$, repetition rate $3 \mathrm{kHz}$ and power rate $\mathrm{P}=123 \mathrm{~mW}$ ), is used in order to cut both the arms and the tips (see Fig. 3).

The actuator consists of a double layer coil (120 windings in total, wire $70 \mu \mathrm{m}$ diameter) wound around a highly oriented crystalline FeSi sheet. Since the easy axis of the FeSi sheet is along its length the core is magnetized longitudinally and 
therefore generating the desired magnetic field for the actuation of the gripper arms (see Fig. 4).

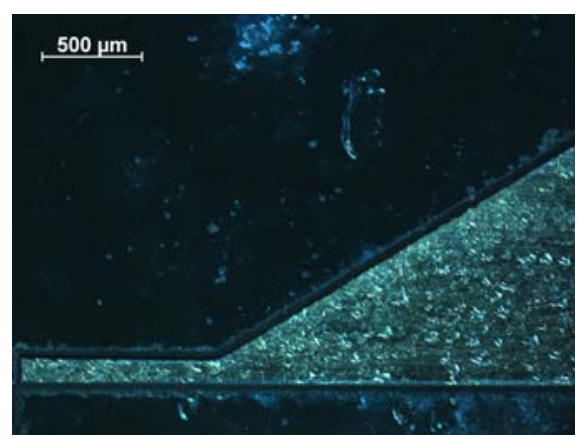

Figure 3: Microgripper tips fabricated from VITROVAC 7505 (soft magnetic, amorphous alloy) using cold laser cutting technique.

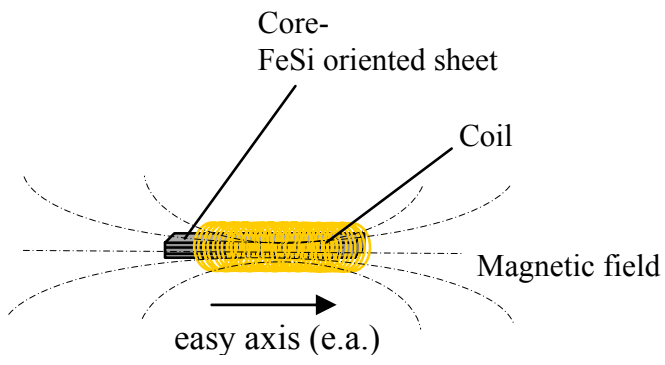

Figure 4: Electromagnetic actuator.

The arms of the microgripper are fixed on the left and right side of the actuator, under a light microscope, by agglutination. The arms of the microgripper are then symmetrically activated by the magnetic field generated by the actuator. Therefore, the opening/closing of both microgripper arms is controlled by the applied DC current (0$500 \mathrm{~mA}$ ). The opening range of the microgripper tips is $0-250$ $\mu \mathrm{m}$ and the normal state of the microgripper is open.

A subminiature LED is integrated on the surface of the microgripper so as to assist the recognition of the microgrippers position from the CCD cameras, as it will be explained in section 2.2. In Figures $5 \mathrm{a}$ and $5 \mathrm{~b}$ the microgripper in open and close state is shown.

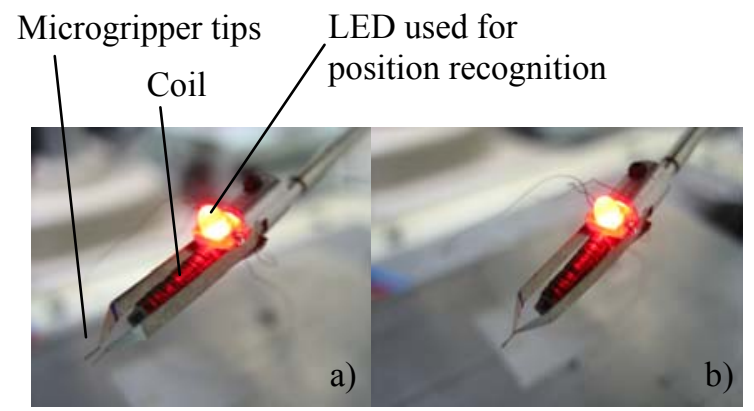

Figure 5a: Magnetic microgripper with open tips. Figure 5b: Magnetic microgripper with closed tips.

\subsection{Visual feedback system}

In order to acquire a position feedback of the robot's endeffector, visual sensors have to be used due to the lack of any integrated position encoders.

In the developed set-up two CCD cameras are used so as to fulfil this requirement. One of the cameras is mounted on the microscope in order to provide sensing of fine positioning. Due to the fact that the scanning field of the optical microscope is rather small, even for the lowest possible magnification, an additional external CCD camera is mounted perpendicular to the robot. This camera overtakes the acquisition of data for approaching the working field in a coarse manner. Furthermore, the final working position in $\mathrm{Z}$ direction has to be reached by this means as fine positioning under the microscope in this direction is not possible.

So as to achieve this goal the position of the gripper has to be obtained with respect to the base of the robot. Therefore a LED is integrated on the microgripper. This LED allows simple, fast and stable position detection.

In particular, the aim is to find a bright spot of the light source (LED) in a rather dark image (see Fig. 6). Therefore, the acquired image is binarized by setting an adequate intensitythreshold value. The resulting image is black, having a white area representing the LED. Furthermore, a particle filter is applied aiming to eliminate possible noise. The center of this white area is then calculated leading to sub-pixel accuracy of the algorithm. The base of the robot is as well easily obtained in $\mathrm{x}$-y image coordinates.

An initial calibration of the camera allows finally the calculation of the real world position of the microgripper in 2 dimensions.

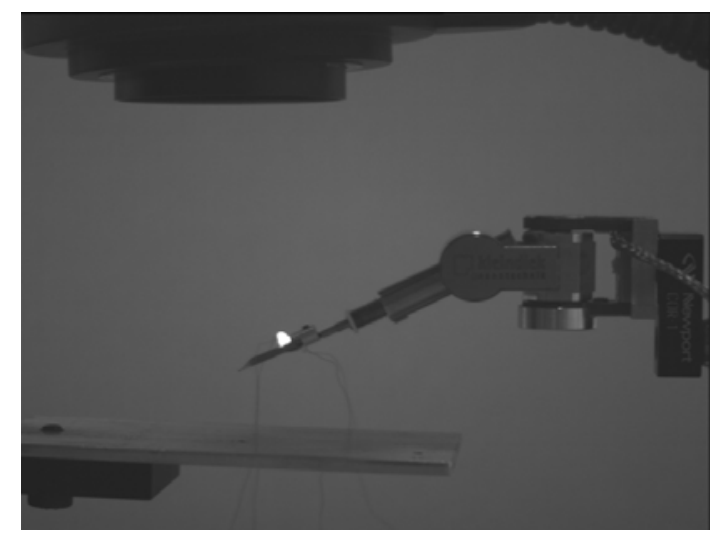

Figure 6: Acquired image by the external CCD camera for coarse positioning. By binarizing the image and applying a particle filter the exact position of the LED can be obtained with sub-pixel accuracy.

\subsection{Implemented control algorithm}

Once the position of the microgripper is known in Cartesian coordinates, inverse kinematics are applied to acquire the actual positions of the robot axes. 
The robot can either be functional in fine mode -when the moment is only based on the elongation or contraction of the robot's driving piezo actuators- or in coarse mode. In the later case, the piezoactuators are operating by the well known "stick-slip" principle. Both modes do not allow the use of classical control theory as motion can only be achieved stepwise.

Nonetheless the term visual servoing is used in this paper as it is not the vision feedback that forces a "LOOK-THEN MOVE" mode but the robot itself.

Another point which complicates the fast and accurate positioning of the robot is the fact that an exact kinematical model for the robot does not exist. This means that the size of the single coarse steps (the mode which is being used in the presented set-up) is not precisely known for none of the three axes. This step-size depends on external forces such as the load being applied to the robot, the cables connected to the microgrippers for their actuation or the direction of the movement. Moreover, environmental influences such as temperature and humidity affect the step size of the robot but to a minor extent.

Taking all the above mentioned into consideration, an adaptive, discrete P- control is implemented (see Fig. 7). As always in closed loop control the input for the control block is the desired position and the fed-back measured position. The actuation parameters for the robot are calculated by means of this difference using a correction factor encoding an estimated size of one step of the robot. This correction factor is updated after every movement of the robot allowing therefore a fast approach of the microgripper to the desired end-position.

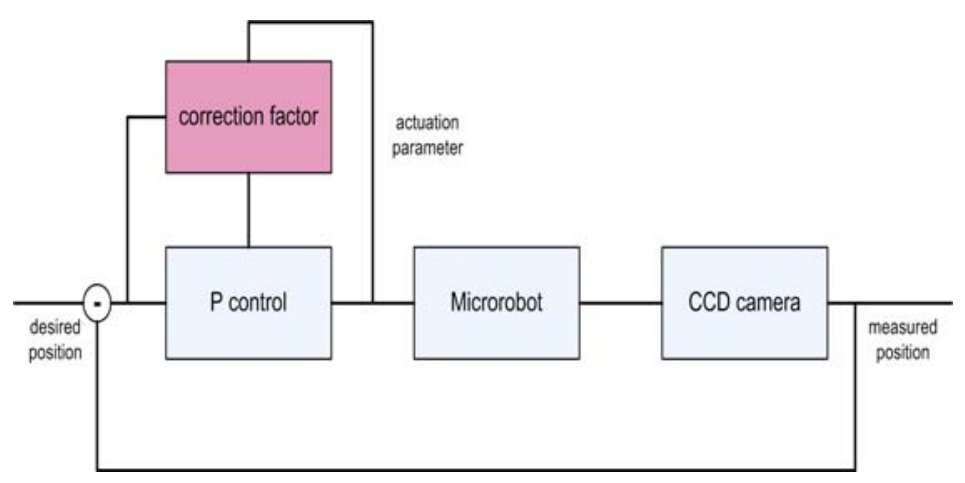

Figure 7: Blockdiagram of the adaptive discrete $\mathrm{P}$ control for the microrobot.

\section{Tests}

In order to characterize the microgripping system and to evaluate its performance, "pick and place" tasks were performed. Optical fibres $(125 \mu \mathrm{m}$ in diameter) were handled in atmospheric conditions. The fibres were placed on the mobile platform perpendicularly to the ground. Figure 8 presents the handling of such a fibre. A ceramic substrate with a V-groove fabricated on it (depth $200 \mu \mathrm{m}$ and width $360 \mu \mathrm{m})$ was also placed on the platform. The micromanipulator moves automatically to the desired position, picks the fibre up and lifts the fibre out of the workspace. Then the mobile platform moves the V-groove to a predefined position underneath the fibre, the micromanipulator moves down and performs the "place" task. Figures $9 \mathrm{a}, 9 \mathrm{~b}, 9 \mathrm{c}$ and $9 \mathrm{~d}$ present the performed task.

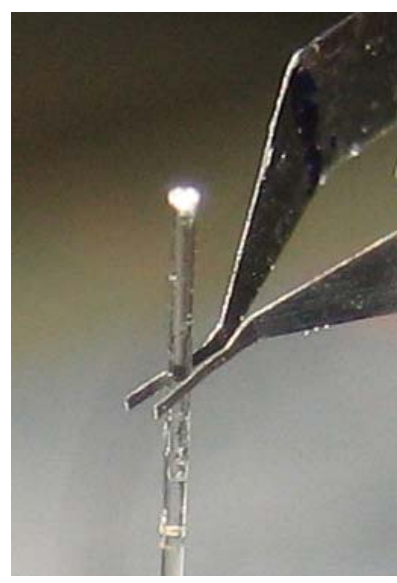

Figure 8: Handling of an optical fibre (125 $\mu \mathrm{m}$ in diameter) using the developed microgripping system.

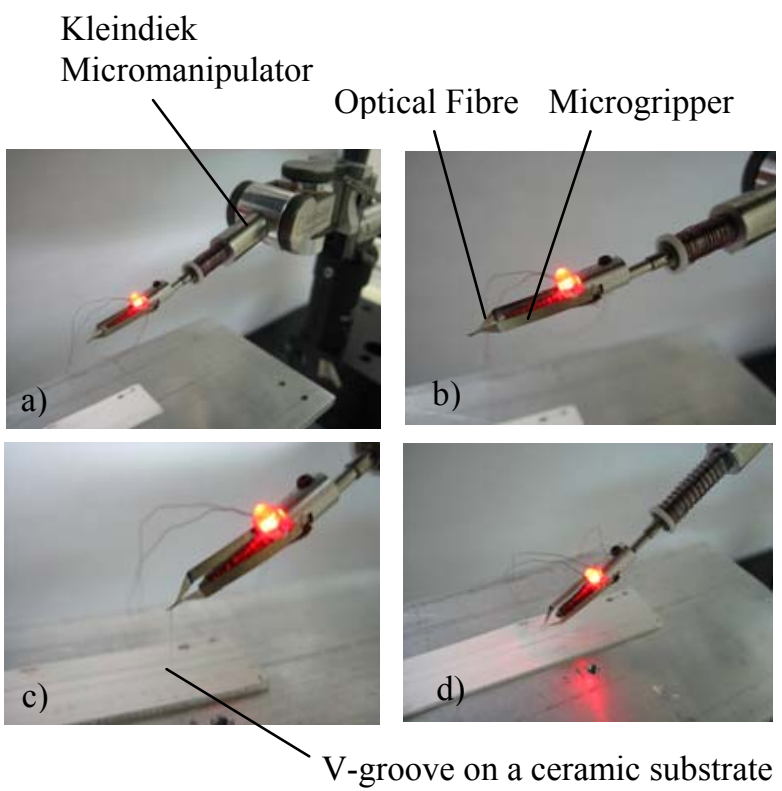

Figure 9a: Positioning of the micromanipulator using the visual feedback.

Figure 9b: "Pick" task of an optical fibre (125 $\mu \mathrm{m}$ in diameter).

Figure 9c: "Place" task of the optical fibre on a V-groove fabricated on a ceramic substrate.

Figure 9d: The optical fibre has been placed on the V-groove. 


\section{Conclusions}

In this paper the development of a flexible, semi-automatic microgripping system consisting of a novel microgripper and a $\mathrm{x}, \mathrm{y}, \mathrm{z}$ positioning system with visual feedback was presented. The novel, low cost, microgripper prototype is fabricated using soft magnetic materials and it is electromagnetically actuated. The function of the commercially available micromanipulator Kleindiek MM3A has been automatized. A subject of future work will be the performance of several case studies, the stereoscopic visual feedback of the whole system as well as the automation of the microgripper's function.

\section{Acknowledgements}

This research activity was funded by the European Commission, FP6, Marie Curie Research Training Network "ASSEMIC".

\section{References}

[1] Sun Lining et. al. "Research on the architecture of virtual reality based on micromanipulation robot", Proc. IEEE conference on Robotics, Intelligent Systems and Signal Processing, volume 2, p. 960, (2003).

[2] S. Fatikow et. al. "Flexible piezoelectric micromanipulation robots for a microassembly desktop station", Proc. $8^{\text {th }}$ International conference on Advanced Robotics, p. 261, (1997).

[3] A. Ferreira. "Automatic microassembly system assisted by vision servoing and virtual reality", IEEE Transactions on Mechatronics, volume 9, p. 321, (2004).

[4] A. Suzuki et. al. "Automated micro handling", Proc. IEEE symposium on Computational Intelligence in Robotics and Automation, volume 1, p. 348, (2003).

[5] Peter. I. Corke. "Visual control of Robots: High performance visual servoing", Mechatronics. Research studies press (John Wiley), (1996).

[6] T. Sano, H. Yamamoto. "Micromanipulation with stereoscopic imaging", IEEE workshop on Imaging Systems and Techniques, p. 48, (2004).

[7] Seok Joo Lee et. al. "Recognizing and tracking of 3Dshaped microparts using multiple visions for micromanipulation", Proc. 2001 international symposium on Micromechatronics and Human Science, , p. 203, (2001).

[8] M. A. Greminger, B. J. Nelson. "Vision based force measurement", IEEE Transactions on Pattern Analysis and Machine Intelligence, volume 26, p. 290, (2004).

[9] Fumihito Arai et. al. "3D micromanipulation system under microscope", Proc. IEEE symposium on Micromechatronics and Human Science, p. 127, (1998).

[10] I. Giouroudi et. al. "Development of a microgripping system for handling of micro components", accepted Proc. $6^{\text {th }}$ international conference of the European society for Precision Engineering and Nanotechnology (Euspen), (2006). 